\title{
Insights Into Particle Transport Obtained from Solar Energetic Particle Anisotropies
}

\author{
R. A. Leske ${ }^{* a}$, A. C. Cummings ${ }^{a}$, C. M. S. Cohen ${ }^{a}$, R. A. Mewaldt ${ }^{a}$, A. W. Labrador ${ }^{a}$, \\ E. C. Stone ${ }^{a}$, M. E. Wiedenbeck ${ }^{b}$, E. R. Christian ${ }^{c}$, and T. T. von Rosenvinge ${ }^{c}$ \\ ${ }^{a}$ California Institute of Technology, Pasadena, CA 91125 USA \\ ${ }^{b}$ Jet Propulsion Laboratory, California Institute of Technology, Pasadena, CA 91109 USA \\ ${ }^{c}$ NASA/Goddard Space Flight Center, Greenbelt, MD 20771 USA \\ E-mail: ral@srl.caltech.edu, ace@srl.caltech.edu, \\ cohendsrl.caltech. edu, rmewaldtesrl.caltech. edu, \\ labrador@srl.caltech.edu, ecs@srl.caltech.edu, \\ Mark.E.Wiedenbeck@jpl.nasa.gov, Eric.R. Christian@nasa.gov, \\ tycho.t.vonrosenvinge@nasa.gov
}

\begin{abstract}
Solar energetic particle (SEP) pitch-angle distributions are shaped by the competing effects of magnetic focusing and scattering as the particles travel through interplanetary space. Therefore, measurements of SEP anisotropies provide insight into particle transport and can probe interplanetary conditions at remote locations from the observer. The Low Energy Telescopes (LETs) onboard the twin STEREO spacecraft measure pitch-angle distributions for protons and heavier ions at energies of about 2-12 MeV/nucleon. Using these instruments, we have observed a wide variety of SEP anisotropies, such as bidirectional flows within interplanetary coronal mass ejections, sunward-flowing particles when the spacecraft was magnetically connected to the back side of a distant shock, and loss-cone distributions in which particles with large pitch angles magnetically mirror at an interplanetary field enhancement that is too weak to reflect particles with the smallest pitch angles. One of the more puzzling observations is unusual oscillations on a timescale of several minutes in the width of a beamed distribution at the onset of the very large 23 July 2012 SEP event. We report LET anisotropy observations at both STEREO spacecraft during the extreme event of 23 July 2012, in which a large range of anisotropies were observed at various times during the event, and discuss their implications for SEP transport.
\end{abstract}

The 34th International Cosmic Ray Conference,

30 July- 6 August, 2015

The Hague, The Netherlands

${ }^{*}$ Speaker. 

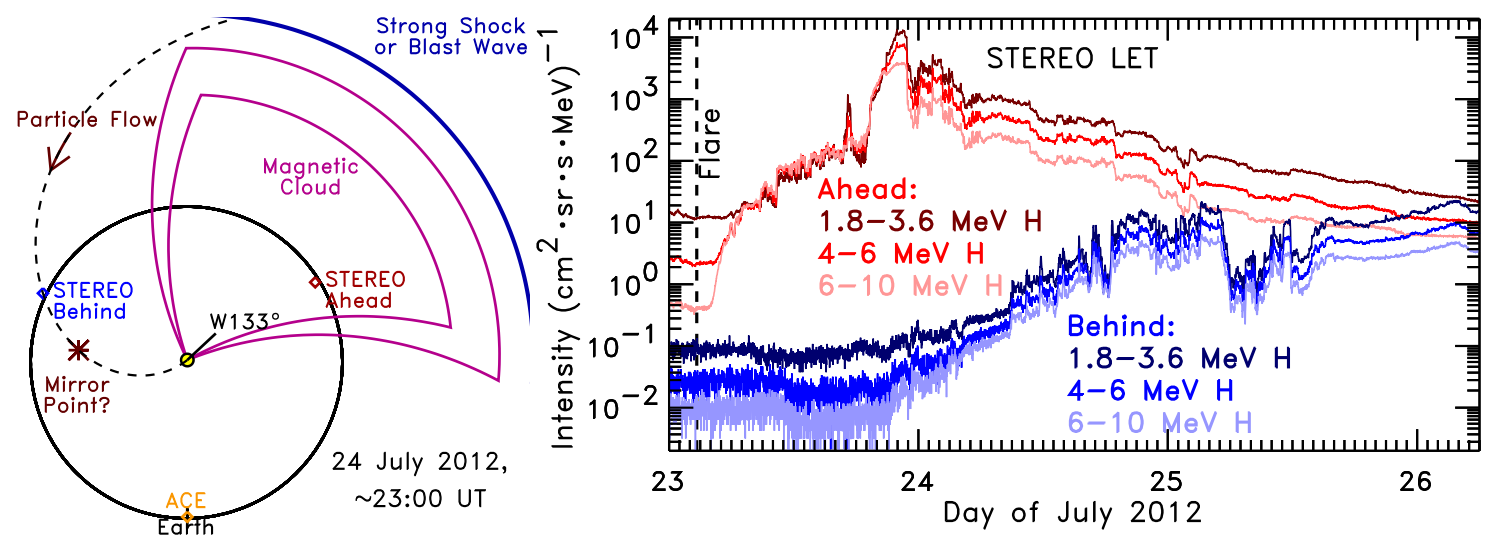

Figure 1: Left: Positions in the ecliptic of Earth and both STEREO spacecraft on 24 July 2012, relative to the approximate location of a strong shock or blast wave driven by a magnetic cloud that erupted the previous day from an active region near $\mathrm{W} 133^{\circ}$. STEREO-Behind is shown connected to the back side of the shock by a nominal field line for a $400 \mathrm{~km} / \mathrm{s}$ solar wind speed (dashed line), however the actual field configuration was far more complicated (see text). The radial spacing of features at the longitude of STEREO-Ahead is approximately correct, but the extent and shape of these features away from this longitude are somewhat speculative and meant for illustration purposes only. Right: Time profiles at 1minute resolution for omnidirectional $\mathrm{H}$ rates in three energy bands from LET on STEREO-Ahead (red) and Behind (blue) during the July 2012 SEP event.

\section{Instrumentation}

Data for this study were collected by the Low Energy Telescopes (LETs) [1] onboard the Solar TErrestrial RElations Observatory (STEREO) spacecraft [2], which measure the composition, spectra, and time variations of solar energetic particles (SEPs) from $\mathrm{H}$ through $\mathrm{Ni}$ at energies of about 2-50 MeV/nucleon. The LET instrument consists of an array of silicon solid-state detectors arranged in two back-to-back fans viewing a range of longitudes in the ecliptic plane, each with a total field of view spanning $133^{\circ}$ of longitude and $\pm 15-20^{\circ}$ of latitude. The fans point $45^{\circ}$ and $-135^{\circ}$ from the Sun (along the nominal Parker spiral field direction), therefore particles in a pair of $47^{\circ}$-wide longitudinal gaps perpendicular to the nominal magnetic field cannot be observed. Each detector is segmented to obtain particle trajectory information, and sectored rates in 16 different longitudinal viewing directions are accumulated onboard for 10 different element and energy bands with angular bins $\sim 12^{\circ}$ wide. During very high count rate periods, the angular coverage for $\mathrm{H}$ and $\mathrm{He}$ is deliberately restricted to reduce electronic deadtime, but heavier elements are still observable over the entire field of view.

\section{Observations}

The largest SEP event detected so far in solar cycle 24 was observed by STEREO-Ahead on 23 July 2012 [3, 4, 5]. As shown in Figure 1, this event originated from an active region over the limb from Earth at $\sim \mathrm{W} 133^{\circ}$ (or near central meridian from the vantage point of Ahead) and was associated with an X-ray flare having an estimated magnitude of M8.2 to X2.5 [6]. At STEREO-Ahead the event included an unusual, particle-mediated blast wave observed in situ at 


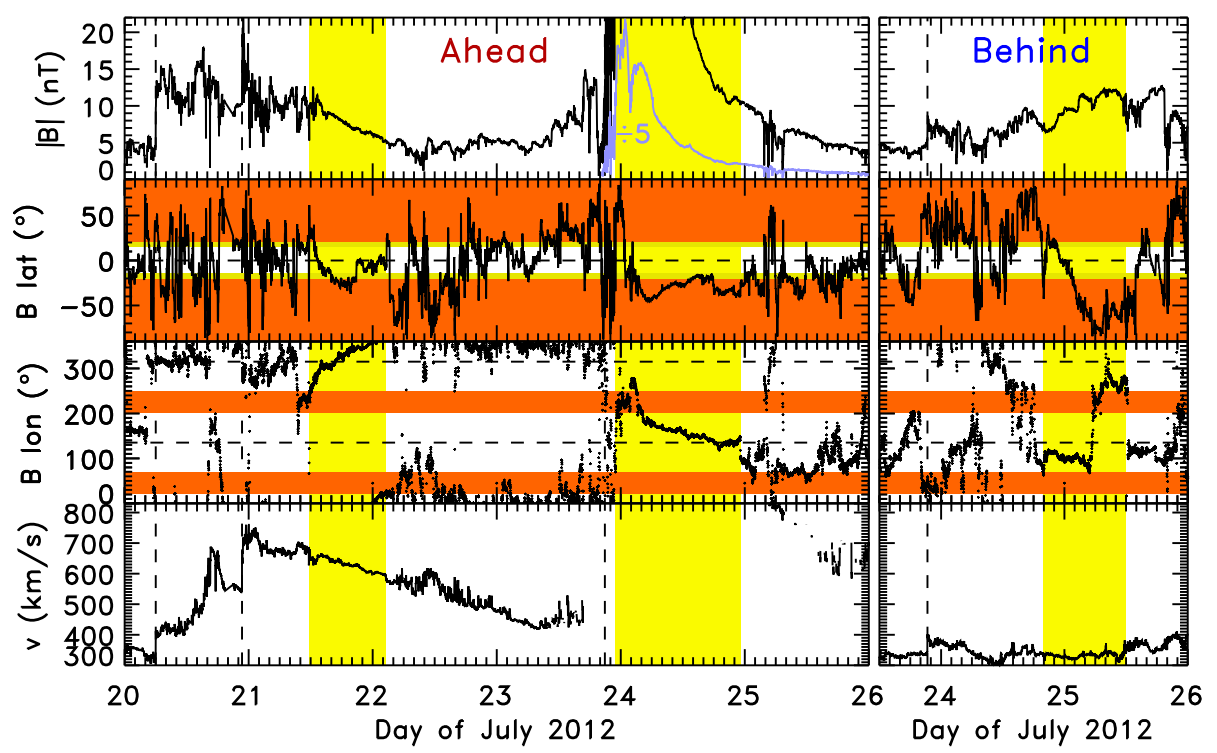

Figure 2: Magnetic field magnitude and direction (in RTN coordinates) from STEREO/MAG and solar wind speed from STEREO/PLASTIC at Ahead (left) and Behind (right). Shocks and ICMEs from http://wwwssc.igpp.ucla.edu/forms/stereo/stereo_level_3.html are shown as vertical dashed lines and yellow shaded regions, respectively. Orange shaded regions indicate directions outside the LET field of view. On 24 July at Ahead the field magnitude is also shown reduced by a factor of 5, and the solar wind proton speed is unavailable throughout most of the day.

speeds exceeding $2000 \mathrm{~km} / \mathrm{s}$ [3]. Only a minor shock was encountered at STEREO-Behind (Figure 2), $124^{\circ}$ of heliolongitude westward from Ahead (Figure 1). Despite the fact that the arrival time of the shock at Behind was nearly the same as that of the very much larger one at Ahead, it is not clear whether this was the same shock or associated instead with one of several other coronal mass ejections (CMEs) during this period [5]. The ICME at Behind, however, had the opposite chirality from that observed at Ahead, and therefore was unrelated [Y. Liu, private communication]. Proton intensities at Ahead were comparable to the highest recorded near Earth since 1972 [4], but while particles were detected at Behind, their intensities were almost 1000 times lower (Figure 1) as the source was beyond the east limb from this perspective. As discussed below, interesting features were seen in the particle anisotropies at both STEREO spacecraft at various times during this event.

An overview of the anisotropies observed by LET during the 2012 July event is shown by the relative intensity spectrograms in Figure 3. Two broad bands, one spanning $-20^{\circ}$ to $110^{\circ}$ from the Sun and the other $160^{\circ}$ to $290^{\circ}$, illustrate particles arriving in the fan pointed $45^{\circ}$ and $-135^{\circ}$ from the Sun, respectively, with each fan comprising 8 sectors. (The white gap between the bands, as well as angles not included on the plot, represent the coverage gaps in ecliptic longitude.) For comparison, the variation in the magnetic field longitude with time measured by STEREO/MAG [7] is also shown. Data plotted for Behind (bottom panel) are sectored rates for 4-6 MeV H, while we instead use 4-6 MeV/nucleon CNO sectored rates for Ahead (top panel) since angular coverage for $\mathrm{H}$ and $\mathrm{He}$ was limited throughout much of the event due to the high count rates.

The most striking feature in Figure 3 at Behind may be seen between $\sim 19: 00$ on 24 July and $\sim 02: 00$ on 25 July. Note that the peak intensity at this time appeared in the fan viewing away from 


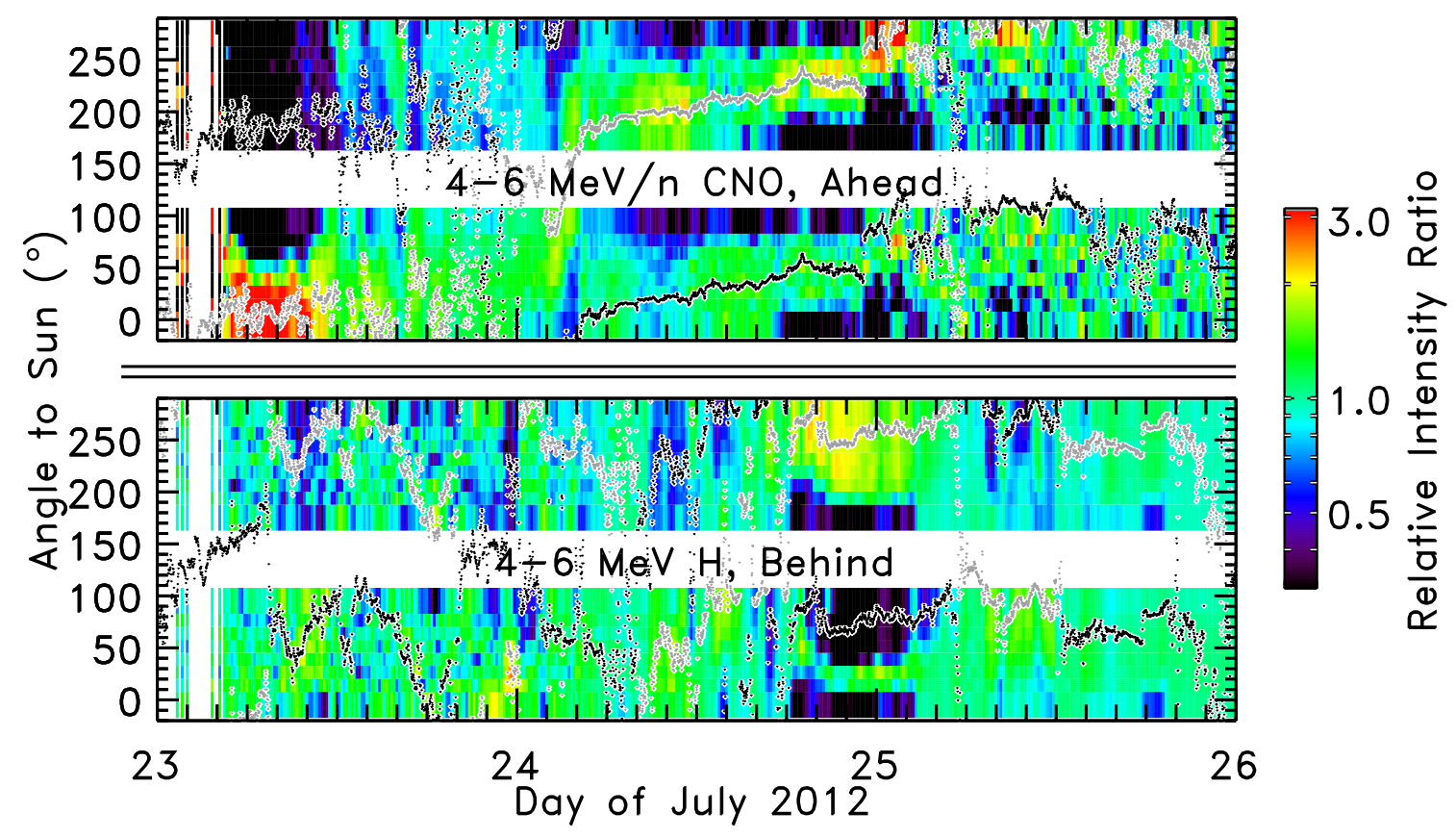

Figure 3: Relative intensities in each of the 16 LET sectors in the in-ecliptic longitudinal viewing directions, for 4-6 MeV/nucleon carbon through oxygen at STEREO-Ahead (top) and 4-6 MeV protons at Behind (bottom) during the July 2012 SEP event. Directions parallel (black) and antiparallel (gray) to the magnetic field longitudes are superposed. The color scale saturates at ratios of $\sim 0.3$ and 3 , but at times the relative intensity ratios were significantly larger (see Figures 4 and 5); colors at all angles would be identical at the value of 1.0 during periods of complete isotropy.

the Sun, that is, the particles were flowing along the field line towards the Sun. Comparison with the solar wind suprathermal electron strahl (which indicates the direction of the field line pointing outward from the Sun [8]) as measured by the Solar Wind Electron Analyzer (SWEA) on STEREO [9] confirms that the electron strahl and LET energetic proton flows were in opposite directions [10] and therefore that the protons were flowing from a location opposite that of the Sun. As illustrated in Figure 1 and suggested by modeling [11], Behind may have been magnetically connected to the back side of the shock, which would be a likely source of these particles. However, it has also been reported that the spacecraft was inside an interplanetary coronal mass ejection (ICME) at this time (see Figure 2). In any case, the particle intensities along the field direction in the fan facing towards the Sun were at a relative minimum during this period, while there was a narrow strip of higher intensities $180^{\circ}$ away from the edge of the incoming beam. This is a loss-cone distribution, which occurs when an incident particle distribution undergoes partial mirroring at an enhanced magnetic field bottleneck [12]. Particles with large pitch angles are reflected, but those with smaller pitch angles pass through the constriction if the field strength is not great enough to turn them around. We have previously described this particular loss-cone distribution in more detail elsewhere $[10,13]$.

At the Ahead spacecraft, throughout most of 24 July (after the shock passage at 20:55 on 23 July), the spacecraft was inside an ICME [5] as indicated in Figure 2. The particle flow was bidirectional at this time (Figure 3), as is often observed in such structures [14], but intensities were higher in the direction facing away from the Sun, suggesting a shorter path to the particle source in 

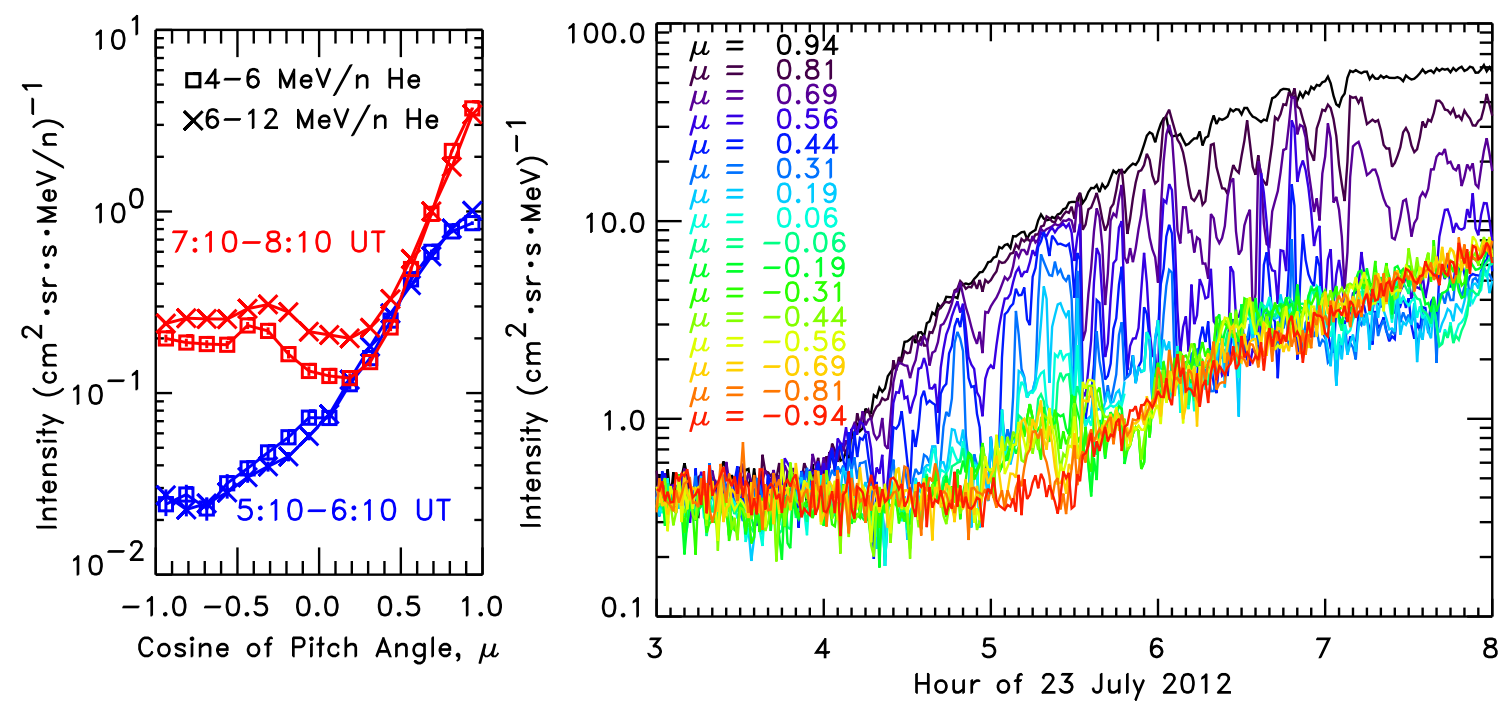

Figure 4: Left: Hourly-averaged pitch-angle distributions for He in two energy bands in LET at STEREOAhead earlier (blue) and later (red) during the onset of the 23 July 2012 event. The distribution appeared narrower later in the event. Right: Time profiles at 1-minute resolution for 6-10 MeV sectored $\mathrm{H}$ in each of 16 pitch-angle bins from LET on STEREO-Ahead at the onset of the 23 July 2012 particle event.

this direction.

Late on 25 July, anisotropies had subsided at both Ahead and Behind (Figure 3). At about this time, proton intensities were nearly the same at both spacecraft (Figure 1), nearly three days after the event onset, suggesting that the particles had isotropized throughout the quasi-trapped, expanding region behind the shock, as is often seen in large SEP events [15].

At the event onset at Ahead, the ions were strongly anisotropic for the first 6 hours. After 08:39 on 23 July, particle intensities at Ahead became large enough that angular coverage was restricted for $\mathrm{H}$ and $\mathrm{He}$, but prior to this their pitch-angle distributions were exponential (Figure 4) and formed a field-aligned, unidirectional beam flowing outwards from the Sun. Curiously, however, the width of the beam appeared to become narrower as the event progressed, at least when examined over longer ( $\sim 1$ hour) timescales. To study this in more detail, in Figure 4 we show intensities in each of 16 bins in $\mu$ (where $\mu$ is the cosine of the pitch angle) vs. time using 1-minute time resolution. In the outward-flowing, field-aligned direction $(\mu=0.94)$, particle intensities rose rather smoothly over 4 hours. The intensity rise was also smooth in the opposite direction $(\mu=-0.94)$, as particles streamed back along the field after an initial delay of $\sim 1.5$ hours. At particle velocities of $\sim 0.11-$ $0.14 c$, this delay corresponds to an outward travel distance of $\sim 0.7 \mathrm{AU}$, suggesting they may have reflected or scattered off the shock that passed Ahead late on 20 July or the ICME of 21 July (see Figure 2). However, for intermediate values of $\mu$ that were not field-aligned, the time profiles were not smooth and the intensities varied tremendously, by factors of 5 or so on timescales of $\sim 10-15$ minutes.

The rapid time variability of the pitch-angle distributions is directly shown by the selection of 1-minute samples in Figure 5. Here it becomes clearer how longer time averages as in Figure 4 might suggest that the beam narrowed with time. Instead, earlier in the event the beam width fluctuated repeatedly by $>25^{\circ}$ in a matter of minutes, while it was much steadier later in the event. 


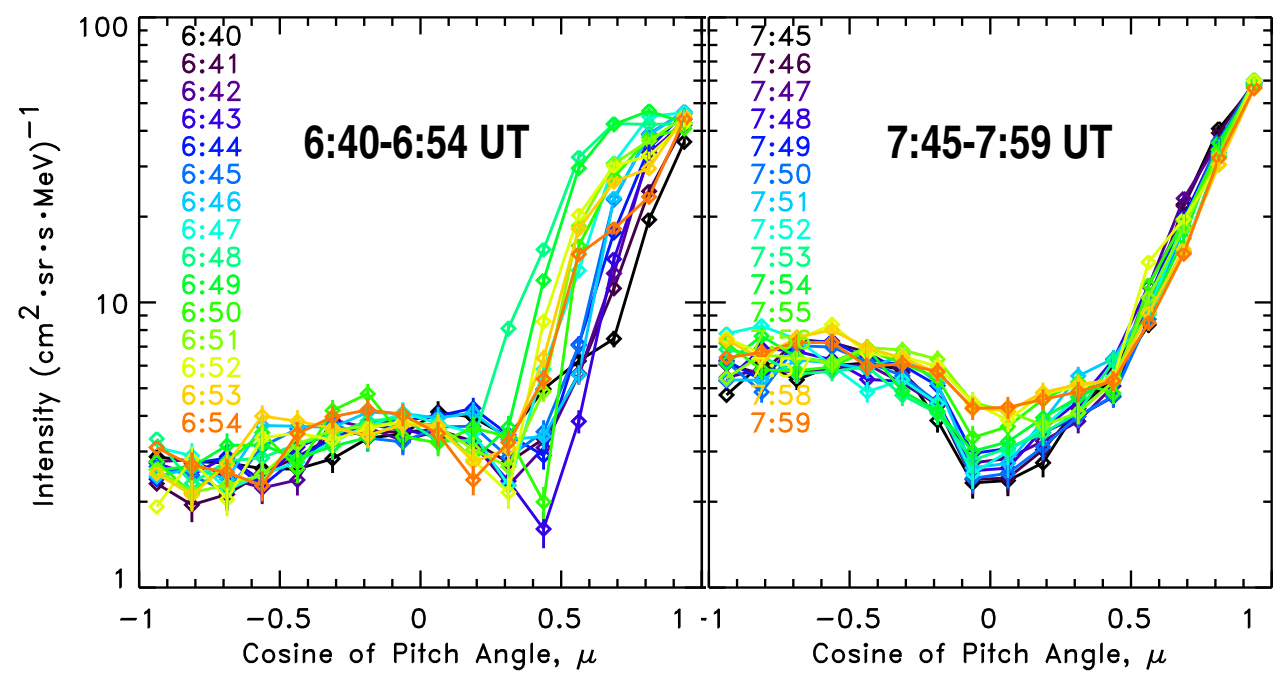

Figure 5: Sample one-minute averaged pitch-angle distributions for 6-10 MeV H in LET at STEREO-Ahead on 23 July 2012 at the times indicated.

Interestingly, even while the width was variable, the slope of the distribution seemed relatively constant, while the peak, field-aligned intensities seemed truncated or suppressed.

\section{Discussion}

Anisotropy measurements can be used to probe heliospheric conditions remote from the observing spacecraft. Within the 23 July event, from two vantage points, we have observed inwardflowing particles, a loss-cone distribution, bidirectional streaming, and a peculiar beam-width oscillation, as described above, which can be used to help understand how the particles become distributed throughout the inner heliosphere.

We have discussed the pronounced loss-cone distribution observed at Behind during the July 2012 event in previous papers $[10,13]$. The width of a loss cone depends on the field strength at the observer and at the mirror point [16, 17], which allows estimation of interplanetary conditions far from the observer. Note that even if the spacecraft was inside of an ICME at this time, the very existence of the loss cone indicates that particles were injected into only one of the ICME legs, with partial mirroring occurring in the other. The fact that the mirror point seems to be located closer to the Sun than the spacecraft requires some explanation. A loss cone would not appear sunward of the spacecraft in an undisturbed Parker spiral field, since eventually the field strength would grow large enough to mirror even the smallest pitch angles. Beyond the magnetic constriction the field must once again have become weaker and allowed the smaller pitch-angle particles through, or scattering must have become more significant. Perhaps interactions of the magnetic cloud with other coronal mass ejections or solar wind streams might have produced the required conditions. Comparison of the anisotropy observations with the results of modeling packages such as the combined WSA-ENLIL-Cone-SEPMOD $[18,19]$ that can predict particle pitch-angle distributions may prove useful.

The oscillations in beam width at the onset of the event at Ahead are puzzling, and to our knowledge such behavior has not been reported before in other events. Note that the field was 
nearly radial for more than a day, from midday on 22 July until the shock arrival late on 23 July (Figure 2), and radial fields appear to be conducive to the propagation of ion cyclotron waves due to reduced refraction and Landau damping in this geometry [20]. Indeed, intermittent and short ion cyclotron waves were observed during the beam-width oscillation period, but they did not appear unusual [L. Jian, private communication]. Later in the day however, from $~ 19: 40-20: 15$, shortly before the arrival of the extreme shock, several small flux ropes with some high frequency magnetic field fluctuations were observed, which is rare [L. Jian, private communication]. If these high frequency fluctuations had been present $\sim 13-16$ hours earlier when we observed the beamwidth oscillations, and if they traveled at the ambient solar wind speed $(\sim 800 \mathrm{~km} / \mathrm{s}$ at 20:00 [3, 5]), they would have been located $\sim 0.27$ AU sunward of the spacecraft at 06:00, and $10 \mathrm{MeV}$ protons would have passed through them only $\sim 15$ minutes before we observed them. More work is needed to determine if the interaction of the particles with these field fluctuations might have produced the unusual anisotropy behavior. Meanwhile, however, it is worth noting that by midday on 23 July particle intensities leveled off within a factor of $\sim 2$ of the streaming limit (Figure 1), which is caused by the scattering of particles by self-generated waves [21]. Although intensities were $\sim 10$ times lower during the oscillations, the restricted range of variability in the field-aligned intensities (Figure 5) might be related to an early phase in the development of the streaming limit.

\section{Acknowledgments}

This work was supported by the National Aeronautics and Space Administration (NASA) at the California Institute of Technology and the Jet Propulstion Laboratory under sub-contract SA2715-26309 from the University of California at Berkeley under NASA contract NAS5-03131, and by NASA award NNX08AK87G. We thank the STEREO/PLASTIC (NASA contract NAS500132), MAG, and SWEA teams for making their data publicly available, and Lan Jian and Ying Liu for helpful discussions.

\section{References}

[1] R. A. Mewaldt, C. M. S. Cohen, W. R. Cook, et al., The Low-Energy Telescope (LET) and SEP Central Electronics for the STEREO Mission, Space Sci. Rev. 136 (Apr., 2008) 285-362.

[2] M. L. Kaiser, T. A. Kucera, J. M. Davila, O. C. St. Cyr, M. Guhathakurta, and E. Christian, The STEREO Mission: An Introduction, Space Sci. Rev. 136 (Apr., 2008) 5-16.

[3] C. T. Russell, R. A. Mewaldt, J. G. Luhmann, et al., The Very Unusual Interplanetary Coronal Mass Ejection of 2012 July 23: A Blast Wave Mediated by Solar Energetic Particles, ApJ 770 (June, 2013) 38.

[4] R. A. Mewaldt, C. T. Russell, C. M. S. Cohen, et al., A $360^{\circ}$ View of Solar Energetic Particle Events, Including One Extreme Event, Proc. 33rd Internat. Cosmic Ray Conf. (Rio de Janeiro) (2013) paper 1186.

[5] Y. D. Liu, J. G. Luhmann, P. Kajdič, et al., Observations of an extreme storm in interplanetary space caused by successive coronal mass ejections, Nature Communications 5 (Mar., 2014) 3481, [arXiv:1405.6088]. 
[6] N. V. Nitta, M. J. Aschwanden, P. F. Boerner, S. L. Freeland, J. R. Lemen, and J.-P. Wuelser, Soft $X$-ray Fluxes of Major Flares Far Behind the Limb as Estimated Using STEREO EUV Images, Solar Phys. (May, 2013) [arXiv:1304.4163].

[7] M. H. Acuña, D. Curtis, J. L. Scheifele, C. T. Russell, P. Schroeder, A. Szabo, and J. G. Luhmann, The STEREO/IMPACT Magnetic Field Experiment, Space Sci. Rev. 136 (Apr., 2008) 203-226.

[8] N. U. Crooker, S. W. Kahler, D. E. Larson, and R. P. Lin, Large-scale magnetic field inversions at sector boundaries, J. Geophys. Res. 109 (Mar., 2004) 3108.

[9] J.-A. Sauvaud, D. Larson, C. Aoustin, et al., The IMPACT Solar Wind Electron Analyzer (SWEA), Space Sci. Rev. 136 (Apr., 2008) 227-239.

[10] R. A. Leske, A. C. Cummings, C. M. S. Cohen, et al., Observations of Loss-Cone Pitch Angle Distributions of Solar Energetic Particles, in Outstanding Problems in Heliophysics: From Coronal Heating to the Edge of the Heliosphere (Q. Hu and G. P. Zank, eds.), vol. 484 of Astronomical Society of the Pacific Conference Series, p. 117, May, 2014.

[11] J. G. Luhmann and D. Odstrcil, Modeling SEP Events in a Forecasting System Context, AGU Meeting of the Americas Abstracts (May, 2013) SH44A-02.

[12] J. W. Bieber, W. Dröge, P. A. Evenson, et al., Energetic Particle Observations during the 2000 July 14 Solar Event, ApJ 567 (Mar., 2002) 622-634.

[13] R. A. Leske, C. M. S. Cohen, R. A. Mewaldt, et al., Solar Energetic Particle Anisotropies Observed by STEREO/LET, Proc. 33rd Internat. Cosmic Ray Conf. (Rio de Janeiro) (2013) paper 0583.

[14] I. G. Richardson, V. M. Dvornikov, V. E. Sdobnov, and H. V. Cane, Bidirectional particle flows at cosmic ray and lower ( $\sim \mathrm{MeV}$ ) energies and their association with interplanetary coronal mass ejections/ejecta, J. Geophys. Res. 105 (June, 2000) 12579-12592.

[15] D. V. Reames, L. M. Barbier, and C. K. Ng, The Spatial Distribution of Particles Accelerated by Coronal Mass Ejection-driven Shocks, ApJ 466 (July, 1996) 473.

[16] K. A. Anderson, J. P. McFadden, and R. P. Lin, Propagation of low energy solar electrons, Geophys. Res. Letters 8 (July, 1981) 831-834.

[17] L. C. Tan, D. V. Reames, C. K. Ng, O. Saloniemi, and L. Wang, Observational Evidence on the Presence of an Outer Reflecting Boundary in Solar Energetic Particle Events, ApJ 701 (Aug., 2009) 1753-1764.

[18] D. Odstrcil, A. Pulkkinen, A. Taktakishvili, P. J. MacNeice, and J. G. Luhmann, Numerical simulation of CMEs and SEPs in the inner heliosphere in August 2010, AGU Fall Meeting Abstracts (Dec., 2011) SH51B-2017.

[19] J. G. Luhmann, S. A. Ledvina, D. Odstrcil, M. J. Owens, X.-P. Zhao, Y. Liu, and P. Riley, Cone model-based SEP event calculations for applications to multipoint observations, Adv. Space Res. 46 (July, 2010) 1-21.

[20] L. K. Jian, C. T. Russell, J. G. Luhmann, R. J. Strangeway, J. S. Leisner, and A. B. Galvin, Ion Cyclotron Waves in the Solar Wind Observed by STEREO Near 1 AU, ApJL 701 (Aug., 2009) L105-L109.

[21] D. V. Reames and C. K. Ng, Streaming-limited Intensities of Solar Energetic Particles, ApJ 504 (Sept., 1998) 1002-1005. 